\title{
A METRIC FORMULA FOR THE GODBILLON-VEY INVARIANT FOR FOLIATIONS
}

\author{
BRUCE L. REINHART AND JOHN W. WOOD ${ }^{1}$
}

\begin{abstract}
The Godbillon-Vey invariant for a foliation of codimension 1 is a cohomology class defined by a 3-form. On a Riemannian manifold, this form can be expressed in terms of the curvature and torsion of the normal curve family and the second fundamental form of the leaves.
\end{abstract}

1. Introduction. Given a foliation $F$ of codimension 1 on a manifold $M$, Godbillon and Vey [1] have defined a de Rham class $g$ in $H^{3}(M ; R)$ which depends only on $F$. If a metric is specified on $M$ then it is possible to make a canonical choice of a 3-form $\gamma$ on $M$ representing $g$. A metric on $M$ also defines a family of normal curves to the foliation and induces a metric structure on the leaves. In this note we describe $\gamma$ in terms of the classical invariants of these curves and immersed submanifolds.

Let $T$ be the vector field on $M$ normal to the foliation (and hence tangent to the normal curves). Let $\kappa$ be the function on $M$ such that $\kappa(x)$ is the curvature of the normal curve at $x$. On the open set where $\kappa \neq 0$, the normal field $N$ and torsion $\tau$ are defined. Let $\omega$ and $\eta$ be the 1-forms dual by the metric to $T$ and $N$. Then

$$
\begin{aligned}
\gamma & =-\kappa^{2} \eta \wedge d \eta & & \text { when } \kappa \neq 0 \\
& =0 & & \text { when } \kappa=0,
\end{aligned}
$$

is a globally defined, smooth form representing the Godbillon-Vey invariant.

Now let $\mathscr{V}$ be an open set where $\kappa \neq 0$, on which there is an orthonormal frame $\left\{T, N, Z_{1}, \cdots, Z_{n-2}\right\}$ with dual frame $\left\{\omega, \eta, \zeta_{1}, \cdots, \zeta_{n-2}\right\}$. Then the Frenet formulas may be written

$$
\begin{gathered}
\nabla_{T} T=\kappa N, \quad \nabla_{T} N=-\kappa T+\sum \tau_{j} Z_{j}, \\
\nabla_{T} Z_{j}=-\tau_{j} N+\sum \alpha_{j k} Z_{k}
\end{gathered}
$$

Received by the editors March 17, 1972.

AMS (MOS) subject classifications (1970). Primary 57D30, 53B20.

Key words and phrases. Godbillon-Vey invariant, Frenet formulas, second fundamental form.

${ }^{1}$ Partially supported by NSF grant GP 7952X3. 
with $\alpha_{j k}$ skew symmetric. Let $l_{T}$ be the second fundamental form of a leaf. Then

$$
\gamma=\kappa^{2} \sum\left(\tau_{j}+l_{T}\left(N, Z_{j}\right)\right) \omega \wedge \eta \wedge \zeta_{j} .
$$

For an oriented 3-manifold this result specializes to a global formula

$$
\gamma=\kappa^{2}\left(\tau+l_{T}(N, Z)\right) * 1
$$

where $* 1$ is the volume form. We prove these formulas in $\S \S 2$ and 3 and calculate some examples on 3-manifolds in $\S 4$.

2. The definition of $\gamma$. Let a foliation on $M$ of codimension 1 be given by a nonvanishing 1 -form $\omega$. Then $d \omega=\omega \wedge \alpha$ for some 1-form $\alpha$, and Godbillon and Vey [1] show that $\gamma=-\alpha \wedge d \alpha$ is a closed form such that its cohomology class $g$ in $H^{3}(M, R)$ depends only on the foliation. Let $M$ be given a Riemannian metric $\langle$,$\rangle with norm | | and covariant derivative \nabla$. Let $T$ be the unit normal field to the foliation, so we may define $\omega$ by $\omega(X)=\langle T, X\rangle$. The curvature $\kappa=\left|\nabla_{T} T\right|$. Let $\mathscr{U}$ be the open set where $\kappa \neq 0$. On $\mathscr{U}$ the normal field $N$ is defined by $\nabla_{T} T=\kappa N$; it follows that $\langle N, T\rangle=0$. Using the definition of $d[3, \mathrm{p} .36]$ and the fact that $\nabla$ is torsion free [3, p. 158], it is easy to check that $d \omega(T, N)=\frac{1}{2} \kappa$. If on some neighborhood where $\kappa \neq 0,\langle Z, T\rangle=\langle Z, N\rangle=0$, then $d \omega(T, Z)=0$. If $\left\langle Z_{i}, T\right\rangle=0$ and $\left\langle Z_{j}, T\right\rangle=0$ then, because $T$ is normal to a foliation, $\left\langle\left[Z_{i}, Z_{j}\right], T\right\rangle=0$. This implies $d \omega\left(Z_{i}, Z_{j}\right)=0$. Define $\eta$ on $\mathscr{U}$ by $\eta(X)=\langle N, X\rangle$. From the definition of $\omega \wedge \eta[3$, p. 35] it follows that $d \omega=\kappa \omega \wedge \eta$. Thus we may take $\alpha=\kappa \eta$. Setting $\alpha=0$ outside of $\mathscr{U}$ gives a smooth, globally defined 1-form. Formula (1) follows from the definition $\gamma=-\alpha \wedge d \alpha$.

If the foliation is not transversely orientable, there is no globally defined nonvanishing $\omega$. However, the unordered pair $\{\omega,-\omega\}$ is defined globally and gives a well-defined $\alpha$ and $\gamma$. Also $\kappa$ and $N$ are independent of the orientation of $T$ along the curves.

Thus the metric enables us to represent the Godbillon-Vey class by a canonical, locally defined form $\gamma$.

3. The relation of $\gamma$ to classical invariants. On the set $\mathscr{U}$ where $\kappa \neq 0$ the form $\kappa \omega \wedge \eta=d \omega$ is a nonzero decomposable, closed 2-form, defining a foliation of codimension 2, each of whose leaves is contained in a leaf of the original foliation. Choose an open set $\mathscr{V}$ and a frame as described in the introduction. Then

$$
d \eta\left(Z_{i}, Z_{j}\right)=-\frac{1}{2} \eta\left(\left[Z_{i}, Z_{j}\right]\right)=-\frac{1}{2}\left\langle N,\left[Z_{i}, Z_{j}\right]\right\rangle=0
$$

because the $Z_{i}$ are tangent to the codimension 2 foliation. Also,

$$
d \eta\left(T, Z_{j}\right)=-\frac{1}{2}\left\langle N, \nabla_{T} Z_{j}\right\rangle+\frac{1}{2}\left\langle N, \nabla_{Z_{j}} T\right\rangle .
$$


Now $l_{T}(X, Y)=\left\langle X, \nabla_{Y} T\right\rangle$ by definition, cf. [2, p. 104]. Hence $d \eta\left(T, Z_{j}\right)=$ $\frac{1}{2} \tau_{j}+\frac{1}{2} l_{T}\left(N, Z_{j}\right)$ and therefore

$$
-\eta \wedge d \eta=\sum_{j=1}^{n-2}\left(\tau_{j}+l_{T}\left(N, Z_{j}\right)\right) \omega \wedge \eta \wedge \zeta_{j}
$$

which implies (2). Note that if $T$ is replaced by $-T$, then $N$ is unchanged but $\tau_{j}$ is replaced by $-\tau_{j}$. Thus the above expression is unchanged, and the formula is applicable to foliations that are not transversely orientable.

For an oriented 3-manifold, $Z$ may be defined on the entire set $\mathscr{U}$ to be the unique vector field such that $T, N, Z$ is an oriented frame field. Then $\omega \wedge \eta \wedge \zeta$ is just the volume element and formula (3) holds on all of $M$ if the function on the right-hand side is defined to be zero when $\kappa=0$.

4. Two examples in dimension three. Consider a foliation of a part of euclidean 3-space whose leaves are surfaces of revolution about a common axis-think of a Reeb component about the $z$-axis. The normal curves lie in planes through the axis so $\tau=0$. Also $N$ lies in these planes so $Z$ is tangent to the meridians. Then $\nabla_{Z} T$ is tangent to the meridians also and $l_{T}(N, Z)=\left\langle N, \nabla_{Z} T\right\rangle=0$. Thus $\gamma \equiv 0$.

For a nonzero example consider $S L(2 ; \boldsymbol{R})$. There is a basis of leftinvariant vector fields $A, B, C$ satisfying $[A, B]=-B,[A, C]=C$, $[B, C]=A$, so $C$ is normal to a foliation. Choose the metric and orientation for which $\{A, B, C\}$ is a positive orthonormal frame. Then:

$$
\nabla_{C} C=A, \quad \nabla_{C} A=-C+\frac{1}{2} B, \quad \nabla_{C} B=-\frac{1}{2} A
$$

as may easily be seen from the formula defining $\nabla$ in terms of the metric [3, p. 160]. Hence $T=C, N=A, Z=B, \kappa=1, \tau=\frac{1}{2}$, and $l_{T}(N, Z)=$ $\left\langle A, \nabla_{B} C\right\rangle=\frac{1}{2}$. Hence $\kappa^{2}(\tau+l)=1$, so $\gamma$ is just the volume element of the group $[1, \S 2] . S L(2 ; R)$ is locally isomorphic to a group of isometries of Lorentz 3-space. Since the hyperbolic plane is isometric to one sheet of the unit hyperboloid in this space, we may restrict the action to this sheet, and thus identify the group with the tangent circle bundle of the hyperbolic plane. The fibers are cosets of the 1-parameter subgroup generated by $2^{-1 / 2}(B+C)$, and make an angle $\pi / 4$ with the foliation. The tangent circle bundle of any orientable 2-manifold of genus greater than one is a compact quotient of this isometry group, and inherits a foliation from the group.

\section{REFERENCES}

1. C. Godbillon and J. Vey, Un invariant des feuilletages de codimension 1, C. R. Acad. Sci. Paris Sér. A-B 273 (1971), A92-A95. MR 44 \#1046.

2. D. Gromoll, W. Klingenberg and W. Meyer, Riemannsche Geometrie im Grossen, 
Lecture Notes in Math., no. 55, Springer-Verlag, Berlin and New York, 1968. MR 37 \#4751.

3. S. Kobayashi and K. Nomizu, Foundations of differential geometry. Vol. I, Interscience, New York, 1963. MR 27 \#2945.

Department of Mathematics, University of Maryland, College Park, MaryLAND 20742 (Current address of B. L. Reinhart)

School of Mathematics, Institute for Advanced Study, Princeton, New JERSEY 08540

Current address (J. W. Wood): Department of Mathematics, Columbia University, New York, New York 10027 